\title{
A note on the properties of power-transformed returns in long-memory stochastic volatility models with leverage effect
}

\author{
Ana Pérez ${ }^{\mathrm{a}}$, Esther Ruiz ${ }^{\mathrm{b}, *}$, Helena Veiga ${ }^{\mathrm{b}}$ \\ a Dpto. Economía Aplicada (Estadística y Econometría), Universidad de Valladolid, Spain \\ ${ }^{\mathrm{b}}$ Dpto. Estadística, Universidad Carlos III de Madrid, Spain
}

\begin{abstract}
A B S T R A C T
The autocorrelation function (acf) of powered absolute returns and their cross-correlations with original returns are derived, for any value of the power parameter, in the context of long-memory stochastic volatility models with leverage effect and Gaussian noises. These autocorrelations and cross-correlations generalize and correct recent results on the acf of squared and absolute returns.
\end{abstract}

\section{Introduction}

An increasingly popular model to represent the dynamic evolution of the volatility of financial returns is the long-memory stochastic volatility model with leverage effect, denoted by A-LMSV model. This model is able to explain the high persistence often observed empirically in the sample autocorrelation function (acf) of squared returns, and the asymmetric response of volatility to positive and negative shocks; see Nakajima and Omori (2008) and Takahashi et al. (2008) for recent results on the importance of asymmetry in stochastic volatility models. On the other hand, in order to characterize adequately the dynamics of conditionally heteroscedastic returns, it is important to know not only the acf of squared returns but also of any power of absolute observations; see, for example, He et al. (2002), Karanasos and Kim (2003, 2006) and Franq and Zakoïan (2008) for references where the autocorrelations of powers of absolute returns are of interest. Furthermore, the presence of an asymmetric response of volatility to positive and negative returns shows up in non-zero cross-correlations between original returns and future powers of absolute returns. Recently, Ruiz and Veiga (2008) derive the properties of the A-LMSV model with Gaussian errors and compare them with those of the FIEGARCH model. In particular, they derive, among other moments, the acf of powers of absolute observations, $\left|y_{t}\right|^{c}$, and the cross-correlations between $y_{t}$ and $\left|y_{t+k}\right|^{c}$, for $c=1$ and 2 , where $c$ is the power parameter. However, the acf in expression (5) of their paper is not valid when $c=1$, i.e. for absolute observations, although it is still valid when $c=2$, i.e. for squared observations. In this note, we derive the expressions of the acf of the power-transformed returns, $\left|y_{t}\right|^{c}$, and of the cross-correlations between $y_{t}$ and $\left|y_{t+k}\right|^{c}$ for any positive power $c>0$. Therefore, we generalize the results in Ruiz and Veiga (2008) allowing a more precise description of the dynamics of returns generated by A-LMSV models. Furthermore, when $c=1$, we obtain, as a particular case, the acf of absolute returns which corrects the wrong expression in Ruiz and Veiga (2008). Finally, when $c=2$, the acf of squared returns comes up and coincides with the expression given in Ruiz and Veiga (2008).

The rest of the note is organized as follows. Section 2 derives the acf and cross-correlations of $\left|y_{t}\right|^{c}$. In Section 3, we obtain the particular cases of the autocorrelations for $c=1$ and 2 . Section 4 concludes the note. 


\section{The acf of power-transformed returns and their cross-correlation with returns}

Consider the Asymmetric Long-Memory Stochastic Volatility, A-LMSV(1, d, 0), model proposed by Ruiz and Veiga (2008), given by

$$
y_{t}=\sigma_{*} \sigma_{t} \varepsilon_{t},
$$

and

$$
(1-\phi L)(1-L)^{d} \log \sigma_{t}^{2}=\eta_{t},
$$

where $y_{t}$ is the return at time $t$ and $\sigma_{*} \sigma_{t}$ is its volatility. The parameter $\sigma_{*}$ is a scale parameter that avoids the inclusion of a constant in the log-volatility equation, (2); see So et al. (1997) for an interpretation of this parameter as the volatility obtained when conditioning upon an average level of information arrival to the market. $L$ is the lag operator such that $L x_{t}=x_{t-1}$. The disturbances $\left(\varepsilon_{t}, \eta_{t+1}\right)^{\prime}$ are assumed to have the following bivariate normal distribution

$$
\left(\begin{array}{c}
\varepsilon_{t} \\
\eta_{t+1}
\end{array}\right) \sim N I D\left[\left(\begin{array}{l}
0 \\
0
\end{array}\right),\left(\begin{array}{cc}
1 & \delta \sigma_{\eta} \\
\delta \sigma_{\eta} & \sigma_{\eta}^{2}
\end{array}\right)\right]
$$

where $\delta$, the correlation between the noises, represents the leverage effect and induces correlation between returns and future volatilities; see Harvey and Shephard (1996). The parameters $\phi$ and $d$ satisfy the stationarity conditions, i.e. $|\phi|<1$ and $|d|<0.5$.

As we mentioned above, the dynamic properties of conditionally heteroscedastic series with leverage effect are reflected in the acf of $\left|y_{t}\right|^{c}$, and in the cross-correlation function between $y_{t}$ and $\left|y_{t+k}\right|^{c}$. Next, we derive both functions for the A-LMSV $(1, d, 0)$ model in (1) and (2).

\subsection{Autocorrelations}

Consider first the autocorrelation of order $k$ of $\left|y_{t}\right|^{c}$ which is given by

$$
\rho_{c}(k)=\frac{\gamma_{c}(k)}{\gamma_{c}(0)}
$$

where $\gamma_{c}(k)=\operatorname{Cov}\left(\left|y_{t}\right|^{c},\left|y_{t+k}\right|^{c}\right)$ and $\gamma_{c}(0)=\operatorname{Var}\left(\left|y_{t}\right|^{c}\right)$. Denote by $h_{t}$ the log-volatility process, i.e. $h_{t}=\log \sigma_{t}^{2}$. Then, the power-transformed returns can be written as

$$
\left|y_{t}\right|^{c}=\sigma_{*}^{c} \exp \left(\frac{c h_{t}}{2}\right)\left|\varepsilon_{t}\right|^{c}
$$

Note that, by definition, $h_{t}$ and $\varepsilon_{t}$ are contemporaneously independent. Therefore, using the properties of the log-normal distribution, the variance of $\left|y_{t}\right|^{c}$ can be derived as follows

$$
\begin{aligned}
\gamma_{c}(0) & =E\left(\left|y_{t}\right|^{2 c}\right)-\left[E\left(\left|y_{t}\right|^{c}\right)\right]^{2} \\
& =\sigma_{*}^{2 c} E\left[\exp \left(c h_{t}\right)\right] E\left(\left|\varepsilon_{t}\right|^{2 c}\right)-\sigma_{*}^{2 c}\left[E\left(\exp \left(\frac{c h_{t}}{2}\right)\right)\right]^{2}\left[E\left(\left|\varepsilon_{t}\right|^{c}\right)\right]^{2} \\
& =\sigma_{*}^{2 c}\left[E\left(\left|\varepsilon_{t}\right|^{c}\right)\right]^{2} \exp \left(\frac{c^{2} \sigma_{h}^{2}}{4}\right)\left[\kappa_{c} \exp \left(\frac{c^{2} \sigma_{h}^{2}}{4}\right)-1\right],
\end{aligned}
$$

where $\kappa_{c}=\frac{E\left(\left|\varepsilon_{t}\right|^{2 c}\right)}{\left[E\left(\left|\varepsilon_{t}\right|^{c}\right)\right]^{2}}$ and $E\left(\left|\varepsilon_{t}\right|^{c}\right)=2^{c / 2} \frac{\Gamma\left(\frac{c}{2}+\frac{1}{2}\right)}{\Gamma\left(\frac{1}{2}\right)}$, where $\Gamma(\cdot)$ is the Gamma function. The expression of $\sigma_{h}^{2}$, the variance of the log-volatility process, which is an $\operatorname{ARFIMA}(1, d, 0)$ process, can be found in Hosking (1981). Note that the expression of $\operatorname{Var}\left(\left|y_{t}\right|^{c}\right)$ given by Ghysels et al. (1996) and subsequently by Ruiz and Veiga (2008) is not correct.

The autocovariance of order $k$ of the power-transformed returns is given by

$$
\begin{aligned}
\gamma_{c}(k) & =E\left(\left|y_{t}\right|^{c}\left|y_{t+k}\right|^{c}\right)-E\left(\left|y_{t}\right|^{c}\right) E\left(\left|y_{t+k}\right|^{c}\right) \\
& =\sigma_{*}^{2 c} E\left(\left|\varepsilon_{t+k}\right|^{c}\right)\left\{E\left[\exp \left(\frac{c\left(h_{t}+h_{t+k}\right)}{2}\right)\left|\varepsilon_{t}\right|^{c}\right]-\exp \left(\frac{c^{2} \sigma_{h}^{2}}{4}\right) E\left(\left|\varepsilon_{t}\right|^{c}\right)\right\} .
\end{aligned}
$$

The first expectation within the squared brackets in (4) cannot be directly decomposed into the product of expectations due to the correlation between $\eta_{t+1}$ and $\varepsilon_{t}$. Consequently, in order to isolate the disturbance $\eta_{t+1}$ in $h_{t}+h_{t+k}$, we consider the following $A R(\infty)$ representation of the log-volatility, $h_{t}=\sum_{i=1}^{\infty} \lambda_{i} \eta_{t+1-i}$, with $\lambda_{1}=1$ and $\lambda_{k}=\sum_{i=0}^{k-1} \frac{\Gamma(i+d)}{\Gamma(i+1) \Gamma(d)} \phi^{k-1-i}$. 
Note that in the short-memory case, when $d=0, \lambda_{k}=\phi^{k-1}$. Then, it turns out that $h_{t}+h_{t+k}-\lambda_{k} \eta_{t+1}$ can be written down as the following linear combination

$$
h_{t}+h_{t+k}-\lambda_{k} \eta_{t+1}=\sum_{i=1}^{k-1} \lambda_{i} \eta_{t+1+k-i}+\sum_{i=1}^{\infty}\left(\lambda_{i}+\lambda_{k+i}\right) \eta_{t+1-i}
$$

which is independent of $\eta_{t+1}$ and Gaussian. Therefore, adding and subtracting $\lambda_{k} \eta_{t+1}$ in the corresponding expectation in (4), we obtain the following result

$$
E\left[\exp \left(\frac{c\left(h_{t}+h_{t+k}\right)}{2}\right)\left|\varepsilon_{t}\right|^{c}\right]=E\left[\exp \left(\frac{c\left(h_{t}+h_{t+k}-\lambda_{k} \eta_{t+1}\right)}{2}\right)\right] E\left[\left|\varepsilon_{t}\right|^{c} \exp \left(\frac{c \lambda_{k} \eta_{t+1}}{2}\right)\right] .
$$

The first expectation in (5) can be obtained by noting that $h_{t}+h_{t+k}-\lambda_{k} \eta_{t+1}$ is normal with zero mean and variance

$$
\operatorname{Var}\left(h_{t}+h_{t+k}-\lambda_{k} \eta_{t+1}\right)=2 \sigma_{h}^{2}\left[1+\rho_{h}(k)\right]-\lambda_{k}^{2} \sigma_{\eta}^{2},
$$

where $\rho_{h}(k)$ is the autocorrelation of order $k$ of the log-squared volatility, $h_{t}$; see Hosking (1981) for the expression of this autocorrelation. Then, using once more the properties of the log-normal distribution, it turns out that

$$
E\left[\exp \left(\frac{c\left(h_{t}+h_{t+k}-\lambda_{k} \eta_{t+1}\right)}{2}\right)\right]=\exp \left(\frac{c^{2} \sigma_{h}^{2}\left[1+\rho_{h}(k)\right]}{4}\right) \exp \left(\frac{-c^{2} \lambda_{k}^{2} \sigma_{\eta}^{2}}{8}\right) .
$$

The second expectation on the right-hand side of (5) can be obtained from Proposition 1 in the Appendix, as follows

$$
E\left[\left|\varepsilon_{t}\right|^{c} \exp \left(\frac{c \lambda_{k} \eta_{t+1}}{2}\right)\right]=\exp \left(\frac{c^{2} \lambda_{k}^{2} \sigma_{\eta}^{2}\left(1-\delta^{2}\right)}{8}\right) \frac{\Gamma(c+1)}{\Gamma\left(\frac{c}{2}+1\right)} 2^{-c / 2} \Phi\left(\frac{c+1}{2}, \frac{1}{2} ; \frac{c^{2} A_{k}^{2}}{2}\right),
$$

where $A_{k}=\frac{\lambda_{k} \delta \sigma_{\eta}}{2}$ and $\Phi(\cdot, \cdot ; \cdot)$ is the degenerate hypergeometric function; see Section 9.21 of Gradshteyn and Ryzhik (1994).

Now, replacing (6) and (7) into (5), putting (5) back into (4) and using formula 8.335.1 of Gradshteyn and Ryzhik (1994), the following expression of the autocovariance of $\left|y_{t}\right|^{c}$ is obtained after some straightforward algebra

$$
\gamma_{c}(k)=\sigma_{*}^{2 c} \exp \left(\frac{c^{2} \sigma_{h}^{2}}{4}\right)\left[E\left(\left|\varepsilon_{t}\right|^{c}\right)\right]^{2}\left[\exp \left(\frac{c^{2} \sigma_{h}^{2} \rho_{h}(k)}{4}\right) \exp \left(-\frac{c^{2} A_{k}^{2}}{2}\right) \Phi\left(\frac{c+1}{2}, \frac{1}{2} ; \frac{c^{2} A_{k}^{2}}{2}\right)-1\right] .
$$

Finally, dividing the autocovariance in (8) by the variance in (3), the expression of the acf of $\left|y_{t}\right|^{c}$ is obtained as follows

$$
\rho_{c}(k)=\frac{\exp \left(\frac{c^{2} \sigma_{h}^{2} \rho_{h}(k)}{4}\right) \exp \left(-\frac{c^{2} A_{k}^{2}}{2}\right) \Phi\left(\frac{c+1}{2}, \frac{1}{2} ; \frac{c^{2} A_{k}^{2}}{2}\right)-1}{\kappa_{c} \exp \left(\frac{c^{2} \sigma_{h}^{2}}{4}\right)-1}, \quad k \geq 1 .
$$

Note that when $d=0$, the acf of the short-memory A-ARSV (1) model is obtained. On the other hand, if there is no leverage effect, i.e. $\delta=0$, then $A_{k}=0$ and $\Phi\left(\frac{c+1}{2}, \frac{1}{2} ; 0\right)=1$. In this case, the acf of the symmetric $\operatorname{LMSV}(1, d, 0)$ model, derived by Harvey (1998), is obtained as a particular case of (9).

\subsection{Cross-correlations}

The cross-correlation of order $k$ between returns and future power-transformed absolute returns is given by

$$
\operatorname{Corr}\left(y_{t},\left|y_{t+k}\right|^{c}\right)=\frac{\operatorname{Cov}\left(y_{t},\left|y_{t+k}\right|^{c}\right)}{\sqrt{\operatorname{Var}\left(y_{t}\right) \operatorname{Var}\left(\left|y_{t+k}\right|^{c}\right)}} .
$$

The variance of $\left|y_{t}\right|^{c}$ has been derived above and it is given by expression (3). On the other hand, the marginal variance of returns is given by

$$
\operatorname{Var}\left(y_{t}\right)=\sigma_{*}^{2} \exp \left(\frac{\sigma_{h}^{2}}{2}\right)
$$

Finally, the cross-covariance between $y_{t}$ and $\left|y_{t+k}\right|^{c}$ can be derived by taking into account that $E\left(y_{t}\right)=0$ and using similar arguments to those used to derive the acf above, as follows

$$
\begin{aligned}
\operatorname{Cov}\left(y_{t},\left|y_{t+k}\right|^{c}\right) & =E\left(y_{t}\left|y_{t+k}\right|^{c}\right)=\sigma_{*}^{c+1} E\left[\exp \left(\frac{h_{t}+c h_{t+k}}{2}\right) \varepsilon_{t}\left|\varepsilon_{t+k}\right|^{c}\right] \\
& =\sigma_{*}^{c+1} E\left(\left|\varepsilon_{t+k}\right|^{c}\right) E\left[\exp \left(\frac{h_{t}+c\left(h_{t+k}-\lambda_{k} \eta_{t+1}\right)}{2}\right)\right] E\left[\varepsilon_{t} \exp \left(\frac{c \lambda_{k} \eta_{t+1}}{2}\right)\right] .
\end{aligned}
$$


To obtain the second expectation in (12), note that $h_{t}+c h_{t+k}-c \lambda_{k} \eta_{t+1}$ is normal with zero mean and variance given by

$$
\operatorname{Var}\left(h_{t}+c h_{t+k}-c \lambda_{k} \eta_{t+1}\right)=\left(1+c^{2}\right) \sigma_{h}^{2}+2 c \sigma_{h}^{2} \rho_{h}(k)-c^{2} \lambda_{k}^{2} \sigma_{\eta}^{2} .
$$

Hence, using the properties of the log-normal distribution, it turns out that

$$
E\left[\exp \left(\frac{h_{t}+c\left(h_{t+k}-\lambda_{k} \eta_{t+1}\right)}{2}\right)\right]=\exp \left(\frac{\sigma_{h}^{2}\left[1+c^{2}+2 c \rho_{h}(k)\right]}{8}\right) \exp \left(\frac{-c^{2} \lambda_{k}^{2} \sigma_{\eta}^{2}}{8}\right) .
$$
by

Now, the last expectation on the right-hand side of (12) is obtained by using Proposition 2 in the Appendix and it is given

$$
E\left[\varepsilon_{t} \exp \left(\frac{c \lambda_{k} \eta_{t+1}}{2}\right)\right]=c A_{k} \exp \left(\frac{c^{2} \lambda_{k}^{2} \sigma_{\eta}^{2}}{8}\right)
$$

where $A_{k}$ is the same as in Section 2.1. Now, putting back (13) and (14) into (12), the following expression for the crosscovariances is obtained

$$
\operatorname{Cov}\left(y_{t},\left|y_{t+k}\right|^{c}\right)=\sigma_{*}^{c+1} E\left(\left|\varepsilon_{t+k}\right|^{c}\right) c A_{k} \exp \left\{\frac{\sigma_{h}^{2}\left[1+c^{2}+2 c \rho_{h}(k)\right]}{8}\right\} .
$$

Finally, replacing (3), (11) and (15) into (10), the order $k$ cross-correlation between $y_{t}$ and $\left|y_{t+k}\right|^{c}$ is given by

$$
\operatorname{Corr}\left(y_{t},\left|y_{t+k}\right|^{c}\right)=\frac{c A_{k} \exp \left(\frac{c \sigma_{h}^{2} \rho_{h}(k)}{4}\right)}{\exp \left(\frac{\sigma_{h}^{2}}{8}\right) \sqrt{\kappa_{c} \exp \left(\frac{c^{2} \sigma_{h}^{2}}{4}\right)-1}}, \quad k \geq 1
$$

see Demos (2002) and Taylor (2005) for the particular case of correlations between returns and future squared returns in the long-memory and short-memory models respectively. Note that formulae (7) and (9) in Ruiz and Veiga (2008) for the cross-covariance and cross-correlation between current returns and future absolute and squared returns, respectively, are immediately obtained as particular cases of (15) and (16) when $c=1$ and $c=2$.

\section{Particular cases: Autocorrelations of absolute and squared returns}

The acf of absolute returns, $\left|y_{t}\right|$, is obtained by taking $c=1$ in (9), as follows

$$
\rho_{1}(k)=\frac{\exp \left(\frac{\sigma_{h}^{2} \rho_{h}(k)}{4}\right) \exp \left(-\frac{A_{k}^{2}}{2}\right) \Phi\left(1, \frac{1}{2} ; \frac{A_{k}^{2}}{2}\right)-1}{\kappa_{1} \exp \left(\frac{\sigma_{h}^{2}}{4}\right)-1}, \quad k \geq 1,
$$

where $\kappa_{1}=\frac{E\left(\varepsilon_{t}^{2}\right)}{\left[E\left(\left|\varepsilon_{t}\right|\right)\right]^{2}}=\frac{\pi}{2}$ and $\Phi\left(1, \frac{1}{2} ; \frac{A_{k}^{2}}{2}\right)=1+\sqrt{\frac{\pi}{2}} A_{k} \exp \left(\frac{A_{k}^{2}}{2}\right)\left[2 \phi\left(A_{k}\right)-1\right]$ with $\phi(\cdot)$ being the cumulative distribution function of the standard normal distribution; see formulae 9.236.1, 9.212.1, 9.212.2 and 8.250.1 in Gradshteyn and Ryzhik (1994). Therefore, the acf of $\left|y_{t}\right|$ is given by

$$
\rho_{1}(k)=\frac{\exp \left(\frac{\sigma_{h}^{2} \rho_{h}(k)}{4}\right)\left\{\exp \left(-\frac{A_{k}^{2}}{2}\right)+\sqrt{\frac{\pi}{2}} A_{k}\left[2 \phi\left(A_{k}\right)-1\right]\right\}-1}{\frac{\pi}{2} \exp \left(\frac{\sigma_{h}^{2}}{4}\right)-1} .
$$

Note that (17) is the correct expression of the autocorrelations of absolute returns in A-LMSV $(1, d, 0)$ models and corrects expression (5) with $c=1$ of Ruiz and Veiga (2008).

Given that it is not easy to derive from (17) any conclusion about the shape of the acf of $\left|y_{t}\right|$, the first column of Fig. 1 plots this acf for the same models chosen in Ruiz and Veiga (2008), namely $\left\{\phi=0, d=0.49, \sigma_{\eta}^{2}=0.05\right\},\{\phi=0, d=$ $\left.0.49, \sigma_{\eta}^{2}=0.1\right\},\left\{\phi=0.5, d=0.49, \sigma_{\eta}^{2}=0.1\right\}$ and $\left\{\phi=0.98, d=0, \sigma_{\eta}^{2}=0.05\right\}$ and $\delta=\{0,-0.2,-0.5,-0.8\}$. Observe that the curves plotted in Fig. 1 for a given model with different values of $\delta$ are hardly distinguishable from each other. Therefore, the presence of leverage effect has a negligible effect on the acf of $\left|y_{t}\right|$. The autocorrelations are nearly the same regardless of the value of the parameter $\delta$ as far as it is negative. Second, we observe that the autocorrelations of $\left|y_{t}\right|$ are always positive and decrease monotonically towards zero from the first lag; compare with the conclusions in Ruiz and Veiga (2008) based on the wrong expression of the acf.

The acf of squared returns, $y_{t}^{2}$, is obtained by taking $c=2$ in (9), as follows

$$
\rho_{2}(k)=\frac{\exp \left(\sigma_{h}^{2} \rho_{h}(k)\right) \exp \left(-2 A_{k}^{2}\right) \Phi\left(\frac{3}{2}, \frac{1}{2} ; 2 A_{k}^{2}\right)-1}{\kappa_{2} \exp \left(\sigma_{h}^{2}\right)-1}, \quad k \geq 1,
$$



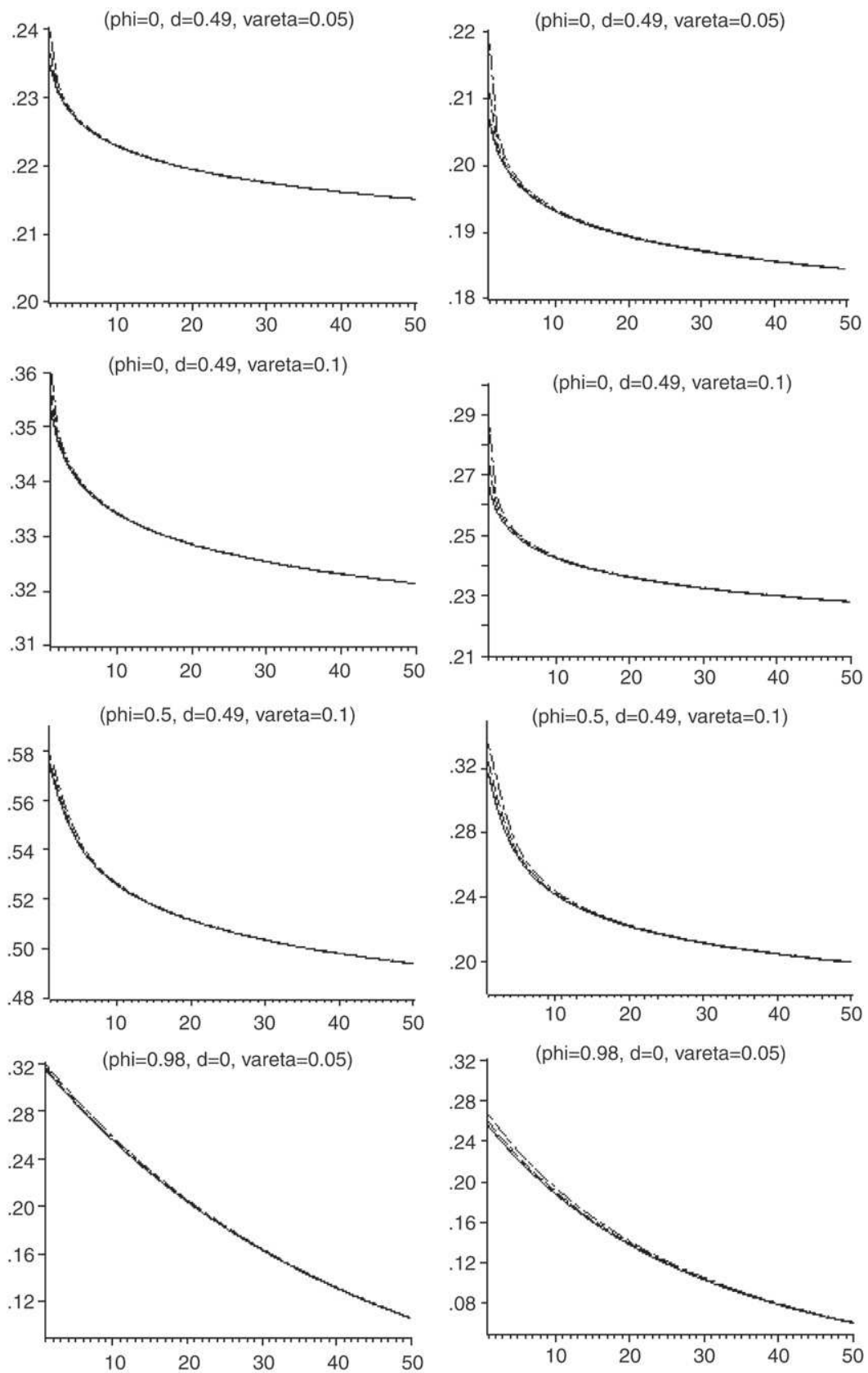

Fig. 1. Autocorrelation function of $\left|y_{t}\right|$ (left column) and $y_{t}^{2}$ (right column) in four A-LMSV models with different values of the asymmetric parameter: continuous $(\delta=0)$, dotted $(\delta=-0.2)$, dashed $(\delta=-0.5)$ and dotted-dashed $(\delta=-0.8)$.

where $\kappa_{2}$ is the kurtosis of $\varepsilon_{t}$, i.e. $\kappa_{2}=3$ and $\Phi\left(\frac{3}{2}, \frac{1}{2} ; 2 A_{k}^{2}\right)=\left(4 A_{k}^{2}+1\right) \exp \left(2 A_{k}^{2}\right)$; see formulae 9.212.2 and 9.215.1 in Gradshteyn and Ryzhik (1994). Therefore, the acf of $y_{t}^{2}$ is given by

$$
\rho_{2}(k)=\frac{\exp \left(\sigma_{h}^{2} \rho_{h}(k)\right)\left(1+\delta^{2} \sigma_{\eta}^{2} \lambda_{k}^{2}\right)-1}{3 \exp \left(\sigma_{h}^{2}\right)-1}, \quad k \geq 1,
$$

which is the same as that reported by Ruiz and Veiga (2008) in their equation 5 when $c=2$. Therefore, their formula (5) is still valid when $c=2$.

Once more, we illustrate the shape of the acf of $y_{t}^{2}$ by plotting in the right column of Fig. 1 the acf's in (18) corresponding to the same models considered above; compare with the autocorrelations of squares of long-memory GARCH models derived by Karanasos et al. (2004). Note that the conclusions are similar to those of the acf of absolute returns. The main difference 

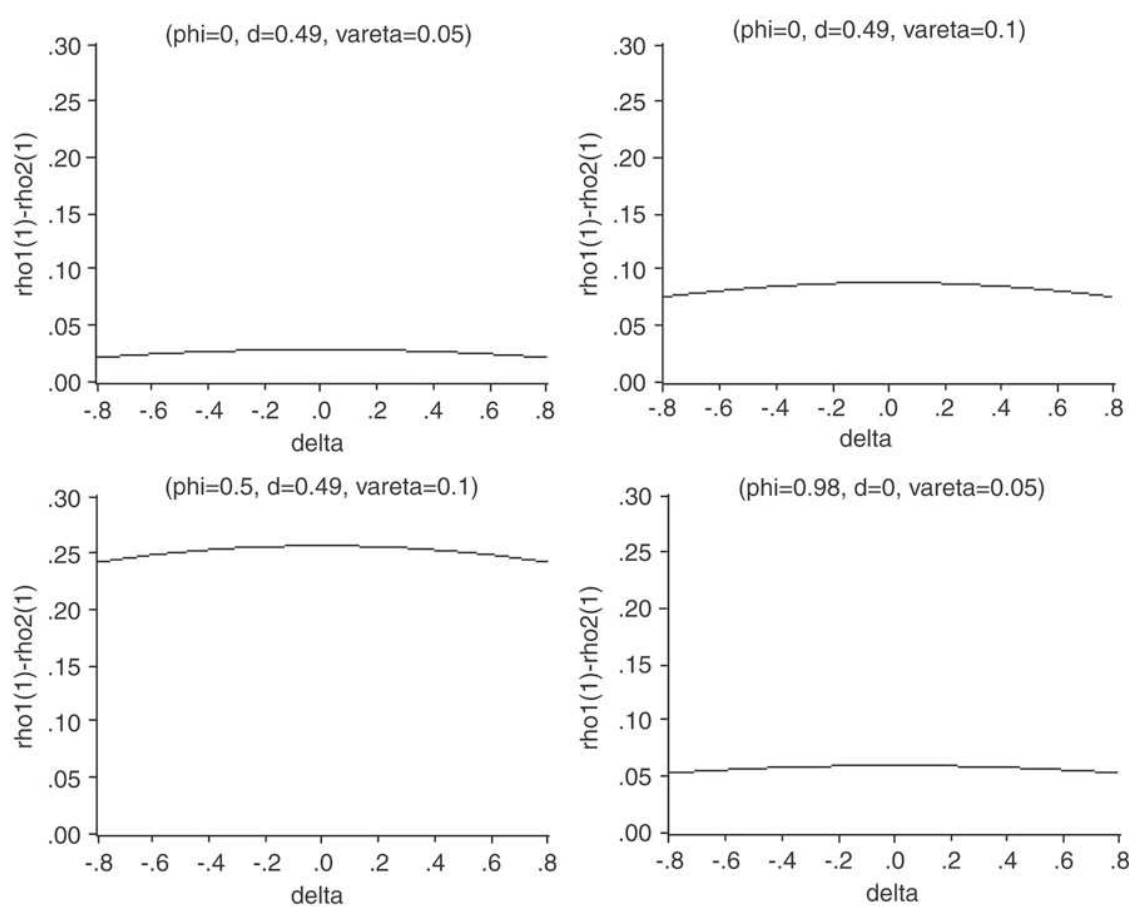

Fig. 2. Differences between the first order autocorrelations of absolute and squared returns as a function of the correlation between $\varepsilon_{t}$ and $\eta_{t+1}$ in four A-LMSV models.

is that the autocorrelations of $y_{t}^{2}$ are systematically smaller than those of $\left|y_{t}\right|$. This phenomenon is known as the Taylor effect in deference to Taylor (1986), who provided extensive empirical evidence on this characteristic of the autocorrelations. To have a clearer picture of this effect in $A-\operatorname{LMSV}(1, d, 0)$ models, Fig. 2 plots, for the same four models mentioned above, the difference between the first order autocorrelations of absolute and squared observations as a function of the asymmetry parameter, $\delta$. This figure shows that these differences are all positive, as postulated by the Taylor effect, and they reach their maximum when there is no leverage effect $(\delta=0)$ and slightly decrease with the absolute value of $\delta$.

\section{Conclusions}

In this note, we extend the results of Ruiz and Veiga (2008) by deriving the autocorrelations of powers of absolute returns and their cross-correlations with the returns themselves, for any value of the power parameter. These functions allow us to have a richer characterization of the dynamics of LMSV models with leverage effect. We also correct a mistake in the expression of the acf of absolute returns given by Ruiz and Veiga (2008) and, consequently, obtain different conclusions on the shape of this acf and on the Taylor effect.

\section{Acknowledgments}

We acknowledge financial support from the Spanish Government, project SEJ2006-03919. The research of A. Pérez was also supported by Junta de Castilla y León, projects VA092A08 and VA027A08. We are very grateful to the editor E. Kontoghiorghes and two anonymous referees for their comments.

\section{Appendix}

Proposition 1. If

$$
\left(\begin{array}{l}
\varepsilon \\
\eta
\end{array}\right) \sim N\left[\left(\begin{array}{l}
0 \\
0
\end{array}\right),\left(\begin{array}{cc}
1 & \delta \sigma_{\eta} \\
\delta \sigma_{\eta} & \sigma_{\eta}^{2}
\end{array}\right)\right],
$$

then for any finite real number a we have that

$$
E\left[|\varepsilon|^{c} \exp (a \eta)\right]=2^{-c / 2} \frac{\Gamma(c+1)}{\Gamma\left(\frac{c}{2}+1\right)} \exp \left(\frac{a^{2} \sigma_{\eta}^{2}\left(1-\delta^{2}\right)}{2}\right) \Phi\left(\frac{c+1}{2}, \frac{1}{2} ; \frac{a^{2} \sigma_{\eta}^{2} \delta^{2}}{2}\right),
$$

where $\Phi(\cdot, \cdot ; \cdot)$ is the degenerate hypergeometric function. 
Proof. From the law of iterated expectations we have that:

$E\left[|\varepsilon|^{c} \exp (a \eta)\right]=E\left(|\varepsilon|^{c} E[\exp (a \eta) \mid \varepsilon]\right)$.

Since the conditional distribution of $\eta$ given $\varepsilon$ is $N\left(\sigma_{\eta} \delta \varepsilon, \sigma_{\eta}^{2}\left(1-\delta^{2}\right)\right)$, using the properties of the log-normal distribution, it turns out that

$$
E[\exp (a \eta) \mid \varepsilon]=\exp \left(a \sigma_{\eta} \delta \varepsilon\right) \exp \left(\frac{a^{2} \sigma_{\eta}^{2}\left(1-\delta^{2}\right)}{2}\right) .
$$

Consequently, plugging this expression into Eq. (20), we have

$$
E\left[|\varepsilon|^{c} \exp (a \eta)\right]=\exp \left(\frac{a^{2} \sigma_{\eta}^{2}\left(1-\delta^{2}\right)}{2}\right) E\left[|\varepsilon|^{c} \exp \left(a \sigma_{\eta} \delta \varepsilon\right)\right] .
$$

Finally, the expectation on the right-hand side of (21) is given by:

$$
\begin{aligned}
E\left[|\varepsilon|^{c} \exp \left(a \sigma_{\eta} \delta \varepsilon\right)\right] & =\frac{1}{\sqrt{2 \pi}} \int_{-\infty}^{\infty}|\varepsilon|^{c} \exp \left(-\frac{\varepsilon^{2}}{2}+a \sigma_{\eta} \delta \varepsilon\right) \mathrm{d} \varepsilon \\
& =\frac{1}{\sqrt{2 \pi}}\left[\int_{0}^{\infty} \varepsilon^{c} \exp \left(-\frac{\varepsilon^{2}}{2}+a \sigma_{\eta} \delta \varepsilon\right) \mathrm{d} \varepsilon+\int_{0}^{\infty} \varepsilon^{c} \exp \left(-\frac{\varepsilon^{2}}{2}-a \sigma_{\eta} \delta \varepsilon\right) \mathrm{d} \varepsilon\right] \\
& =\frac{1}{\sqrt{2 \pi}} \Gamma(c+1) \exp \left(\frac{a^{2} \sigma_{\eta}^{2} \delta^{2}}{4}\right)\left[D_{-(c+1)}\left(-a \sigma_{\eta} \delta\right)+D_{-(c+1)}\left(a \sigma_{\eta} \delta\right)\right],
\end{aligned}
$$

where D.(.) is the parabolic cylinder function; see formula 3.462.1 in Gradshteyn and Ryzhik (1994). Now, using formula 9.240 in Gradshteyn and Ryzhik (1994), it can be proved that

$$
D_{-(c+1)}\left(-a \sigma_{\eta} \delta\right)+D_{-(c+1)}\left(a \sigma_{\eta} \delta\right)=\frac{2^{-c / 2} \sqrt{2 \pi}}{\Gamma\left(\frac{c}{2}+1\right)} \exp \left(-\frac{a^{2} \sigma_{\eta}^{2} \delta^{2}}{4}\right) \Phi\left(\frac{c+1}{2}, \frac{1}{2} ; \frac{a^{2} \sigma_{\eta}^{2} \delta^{2}}{2}\right) .
$$

Then, replacing this expression into Eq. (22) it turns out that

$$
E\left[|\varepsilon|^{c} \exp \left(a \sigma_{\eta} \delta \varepsilon\right)\right]=2^{-c / 2} \frac{\Gamma(c+1)}{\Gamma\left(\frac{c}{2}+1\right)} \Phi\left(\frac{c+1}{2}, \frac{1}{2} ; \frac{a^{2} \sigma_{\eta}^{2} \delta^{2}}{2}\right),
$$

and putting back this value into (21) the result in (19) comes up.

Proposition 2. If

$$
\left(\begin{array}{l}
\varepsilon \\
\eta
\end{array}\right) \sim N\left[\left(\begin{array}{l}
0 \\
0
\end{array}\right),\left(\begin{array}{cc}
1 & \delta \sigma_{\eta} \\
\delta \sigma_{\eta} & \sigma_{\eta}^{2}
\end{array}\right)\right],
$$

then for any finite real number a we have that

$$
E[\varepsilon \exp (a \eta)]=a \delta \sigma_{\eta} \exp \left(\frac{a^{2} \sigma_{\eta}^{2}}{2}\right) .
$$

Proof. The conditional distribution of $\eta$ given $\varepsilon$ is $N\left(\sigma_{\eta} \delta \varepsilon, \sigma_{\eta}^{2}\left(1-\delta^{2}\right)\right)$. Therefore by the law of iterated expectations and the properties of the log-normal distribution, we obtained the following result

$$
\begin{aligned}
E[\varepsilon \exp (a \eta)] & =E[\varepsilon E(\exp (a \eta) \mid \varepsilon)] \\
& =\exp \left\{\frac{a^{2} \sigma_{\eta}^{2}\left(1-\delta^{2}\right)}{2}\right\} E\left[\varepsilon \exp \left(a \delta \sigma_{\eta} \varepsilon\right)\right] .
\end{aligned}
$$

The expectation on the right-hand side of (24) is given by

$$
\begin{aligned}
E\left[\varepsilon \exp \left(a \delta \sigma_{\eta} \varepsilon\right)\right] & =\frac{1}{\sqrt{2 \pi}} \int_{-\infty}^{\infty} \varepsilon \exp \left(-\frac{\varepsilon^{2}}{2}+a \delta \sigma_{\eta} \varepsilon\right) \mathrm{d} \varepsilon \\
& =a \delta \sigma_{\eta} \exp \left(\frac{a^{2} \delta^{2} \sigma_{\eta}^{2}}{2}\right) ;
\end{aligned}
$$

see formula 3.462.6 in Gradshteyn and Ryzhik (1994).

Finally, replacing (25) into (24), the result in (23) is obtained. 


\section{References}

Demos, A., 2002. Moments and dynamic structure of a time varying parameter stochastic volatility in mean model. Econometrics Journal $5,345-357$.

Franq, C., Zakoïan, J., 2008. Deriving the autocovariances of powers of markov-switching GARCH models, with applications to statistical inference. Computational Statistics \& Data Analysis 52 (4), 3027-3046.

Ghysels, E., Harvey, A., Renault, E., 1996. Stochastic volatility. In: Rao, C.R., Maddala, G. (Eds.), Statistical Methods in Finance. North-Holland, Amsterdam, pp. 119-191.

Gradshteyn, I., Ryzhik, I., 1994. Table of Integrals, Series and Products. Academic Press.

Harvey, A., Shephard, N., 1996. Estimation of an asymmetric stochastic volatility model for asset returns. Journal of Business and Economic Statistics 14 429-434.

Harvey, A.C., 1998. Long-memory in stochastic volatility. In: Knight, J., Satchell, S. (Eds.), Forecasting Volatility in Financial Markets. ButterworthHeinemann, London.

He, C., Teräsvirta, T., Malmsten, H., 2002. Fourth moment structure of a family of first-order exponential GARCH models. Econometric Theory 18 , $868-885$. Hosking, J., 1981. Fractional differencing. Biometrika 68, 165-176.

Karanasos, M., Kim, J., 2003. Moments of the ARMA-EGARCH model. Econometrics Journal 6 (1), 146-166.

Karanasos, M., Kim, J., 2006. A re-examination of the asymmetric power ARCH model. Journal of Empirical Finance 13, 113-128.

Karanasos, M., Psaradakis, Z., Sola, M., 2004. On the autocorrelation properties of long-memory GARCH processes. Journal of Time Series Analysis 25 (2), 265-281.

Nakajima, J., Omori, Y., 2008. Leverage, heavy-tails and correlated jumps in stochastic volatility models. Computational Statistics \& Data Analysis, doi:10.1016/j.csda.2008.03.015.

Ruiz, E., Veiga, H., 2008. Modelling long-memory volatilities with leverage effect: A-LMSV versus FIEGARCH. Computational Statistics \& Data Analysis 52 (6), 2846-2862.

So, M., Lam, K., Li, W., 1997. An empirical study of volatility in seven southeast asian stock markets using ARV models. Journal of Business Finance \& Accounting $24(2), 261-275$

Takahashi, M., Omori, Y., Watanabe, T., 2008. Estimating stochastic volatility models using daily returns and realized volatility simultaneously. Computational Statistics \& Data Analysis, doi:10.1016/j.csda.2008.07.39.

Taylor, S., 1986. Modelling Financial Time Series. Wiley: New York.

Taylor, S., 2005. Asset Price Dynamics, Volatility and Prediction. Princeton University Press. 